\title{
Method for assessment of laboratory turnround times: Comparison before, during, and after analysis
}

\author{
W S A Smellie, J Johnston, P J Galloway
}

\begin{abstract}
Aims-To establish a mechanism to examine the components of turnround time in a representative cross-section of laboratory users; and to identify potential areas for improvement.
\end{abstract}

Methods-Information was collected manually from result reports received by eight laboratory users: three wards in the main hospital, four GP practices, and one local psychiatric hospital. This was combined with data from the departmental computer files to create a spreadsheet detailing different time points in the processing of a specimen, from venepuncture to receipt of result report.

Results-At the main hospital, $80 \%$ of samples arrived within two hours of venesection and $95 \%$ by four hours; $75 \%$ of samples were analysed within two hours; $85 \%$ of results arrived in the wards within six hours of printing, although $12 \%$ took more than 18 hours to arrive; median overall time six hours. At the satellite (psychiatric) hospital, all samples arrived within seven hours of venesection; $45 \%$ were analysed within two hours-the rest the following morning; there were highly variable post-analytical times, minimum 18 hours, maximum 122 hours; the median overall time was 69 hours. Twenty five per cent of samples from GPs took more than 20 hours to arrive; $75 \%$ were analysed within two hours, the rest took over 18 hours-waiting overnight; the post-analytical times were highly variable, minimum 22 hours, maximum 122 hours; the median overall time was 50 hours.

Conclusions-The method is easily repeatable and demonstrates the need for local improvement in the post-analytical period. Although specific to the individual data handling system for one laboratory, this method may be used as a basis for other laboratories in pathology disciplines to undertake a representative assessment of turnround times for different groups of laboratory users.

$(F$ Clin Pathol 1994;47:585-588)
"Auditors found that slow turnaround times for specimens and reports were not uncommon, but were rarely the result of slow work in the laboratory. They were usually caused by poor arrangements for collecting specimens or returning results. Often these arrangements were not managed by laboratories themselves but they were invariably blamed when things went wrong."

These findings from the Audit Commission, which has recently published recommendations and proposed methods for improving laboratory turnround, indicate the importance of pre- and post-analytical periods in laboratory management. One recent audit in a microbiology laboratory corroborates these findings. ${ }^{2}$ About $20 \%$ of the core analytical workload at Glasgow Royal Infirmary originates from local general practitioners and outlying hospitals, although laboratory figures available for Scotland vary greatly between laboratories and general practice work may exceed $50 \% .^{3}$ We have designed a protocol for assessing turnround times which will allow the component parts of total turnround to be separated and compared.

A pilot study (unpublished) of turnround times was performed in 1992 in conjunction with staff at Lightburn Geriatric Hospital, Glasgow, to identify the time periods between venepuncture and result reports being received by the requesting clinician. This had shown Lightburn post-analytical times to be up to five days over weekends and led to an interim solution with an increase in faxing and telephoning of results.

\section{Methods}

The Lightburn study was extended and applied throughout the laboratory's catchment area in July 1993. All samples from the outlying practices and hospital sites selected are delivered to the Royal Infirmary once a day by van delivery. All results are posted out using the hospital internal mail collection system, and thence via the Royal Mail to general practices, or by a twice daily van service (departing at 11.00 am and $2.00 \mathrm{pm}$ ) to the outlying hospitals. All external mail, including reports, for posting via the hospital mail system is delivered to the central mailing office
J Johnston

P J Galloway

Correspondence to:

Accepted for publication 11 January 1994 
Ward/Unit:

\begin{tabular}{|c|c|cc|}
\hline $\begin{array}{c}\text { ACCESSION } \\
\text { NUMBER }\end{array}$ & $\begin{array}{c}\text { FORM } \\
\text { TYPE } \\
\text { A or B }\end{array}$ & \multicolumn{2}{|c|}{$\begin{array}{c}\text { DATE/TIME } \\
\text { RECEIVED }\end{array}$} \\
\hline $105683 X$ & A & $9.30 \mathrm{am}$ & $06 / 09 / 93$ \\
\hline & & & \\
\hline & & & \\
\hline & & \\
\hline & & & \\
\hline
\end{tabular}

Figure 1 Log sheet for recording arrival times of reports.

by the department porter twice daily at about 9.30 am and $2.00 \mathrm{pm}$.

\section{STUDY DESIGN}

(1)-Representative target units were selected to include wards within Glasgow Royal Infirmary, one satellite hospital (psychiatric unit), and four general practices (two multiple practice health centres and two single practice centres). Three sites were selected within Glasgow Royal Infirmary as being representative of the local geography of the hospital.

(2)-Clinical, nursing, and clerical staff in the target units were approached informally to enlist support for the scheme and to encourage participation.

(3) - A study period of between one and three weeks was chosen, depending on the unit, as we required 50 samples from each site to permit valid statistical analysis of results.

(4) - During the study period, medical staff were asked to record sample withdrawal times on all forms sent with samples for analyses to the laboratory.

(5)-Clerical staff in target units were requested to record the date and precise time that a result report was received at its destination, together with the laboratory sample identification number (accession number) and the result type (form type A: serum urea and electrolytes, osmolalities, glucose and amylase; form type B: calcium, phosphate, and liver function tests). Specific log sheets were provided for this purpose (fig 1).

(6) - Specially created software, described below, was used to access time data retrospectively on samples documented by clerical staff at participating sites.

\section{COMPUTING}

Two files for each form type were accessed on the departmental DEC PDP 11/44 mini-computer. The first file is used to record laboratory accession number and the date and time of the first report produced by the computer for that sample-regardless of whether or not all analyses have been performed. The time of production of subsequent complete reports is not recorded.

The second, an archive file, contains patient demographic information, laboratory accession number, date and time the sample was withdrawn as recorded on the request form, and the date and time received in the laboratory. This information is all entered initially in the computer when samples are logged before analysis.

A third file was created using data from the specific log sheets (fig 1) returned from all the relevant sites.

The laboratory accession number of the sample was used to match entries in the three files and to link the following data for each sample: date and time sample was withdrawn; date and time sample was received by the laboratory; date and time the first report was produced; date and time the report was received by the ward. All times were recorded to the nearest hour.

The times taken for each component of turnround were calculated. The data were then analysed by frequency distribution.

\section{SPECIFIC PROBLEMS}

(1)-Accurate timing of withdrawal

For the purposes of this study, withdrawal time was taken to be the time recorded on the request form by the person performing venepuncture. No attempt was made to record the precise time at which a sample left the ward or practice because of the administrative difficulty involved.

(2)-Accurate recording of time of specimen receipt

Specimen receipt times were deemed to be the time stamped on request forms on receipt in the sample reception area. This time, however, may be after a large batch of samples had been separated in reception.

(3)-No sample withdrawal time given

If a sample withdrawal time was not recorded on the request form clerical staff entered a time identical with that of specimen reception. Sample withdrawal and specimen reception times therefore were identical in the computer file, indicating that no sample withdrawal time has been given, and the pre-analytical time was excluded from analysis.

(4)-Delays in a report reaching the target unit A possible reason for increased turnround time is delay in opening envelopes containing results at the ward or practice. We endeavoured to avoid these delays by marking envelopes containing study results with a coloured stamp to facilitate recognition. This stamp was not identifiable by portering or mailroom staff and therefore should not have interfered with the normal transportation time incurred in reaching target units, but should have allowed clerical staff to recognise easily study envelopes on receipt. 
Median values and ranges for pre-, peri-and post-analytical periods (hours) for the base hospital (Parkhead Hospital) and four general practices

\begin{tabular}{llll}
\hline Time/site & Hospital wards & General practitioners & Satellite hospital \\
\hline Pre-analytical & Median 1 & Median 5 & Median 5 \\
& Range 1-27 & Range 1-71 & Range 3-7 \\
& $\mathrm{n}=117$ & $\mathrm{n}=150$ & $\mathrm{n}=50$ \\
Analytical & Median 1 & Median 1 & Median 20 \\
& Range 1-26 & Range 1-92 & Range 1-68 \\
& $\mathrm{n}=193$ & $\mathrm{n}=166$ & $\mathrm{n}=77$ \\
Post-analytical & Median 4 & Median 44 & Median 44 \\
& Range 1-48 & Range 24-120 & Range 16->120 \\
& $\mathrm{n}=193$ & $\mathrm{n}=166$ & $\mathrm{n}=77$ \\
\hline
\end{tabular}
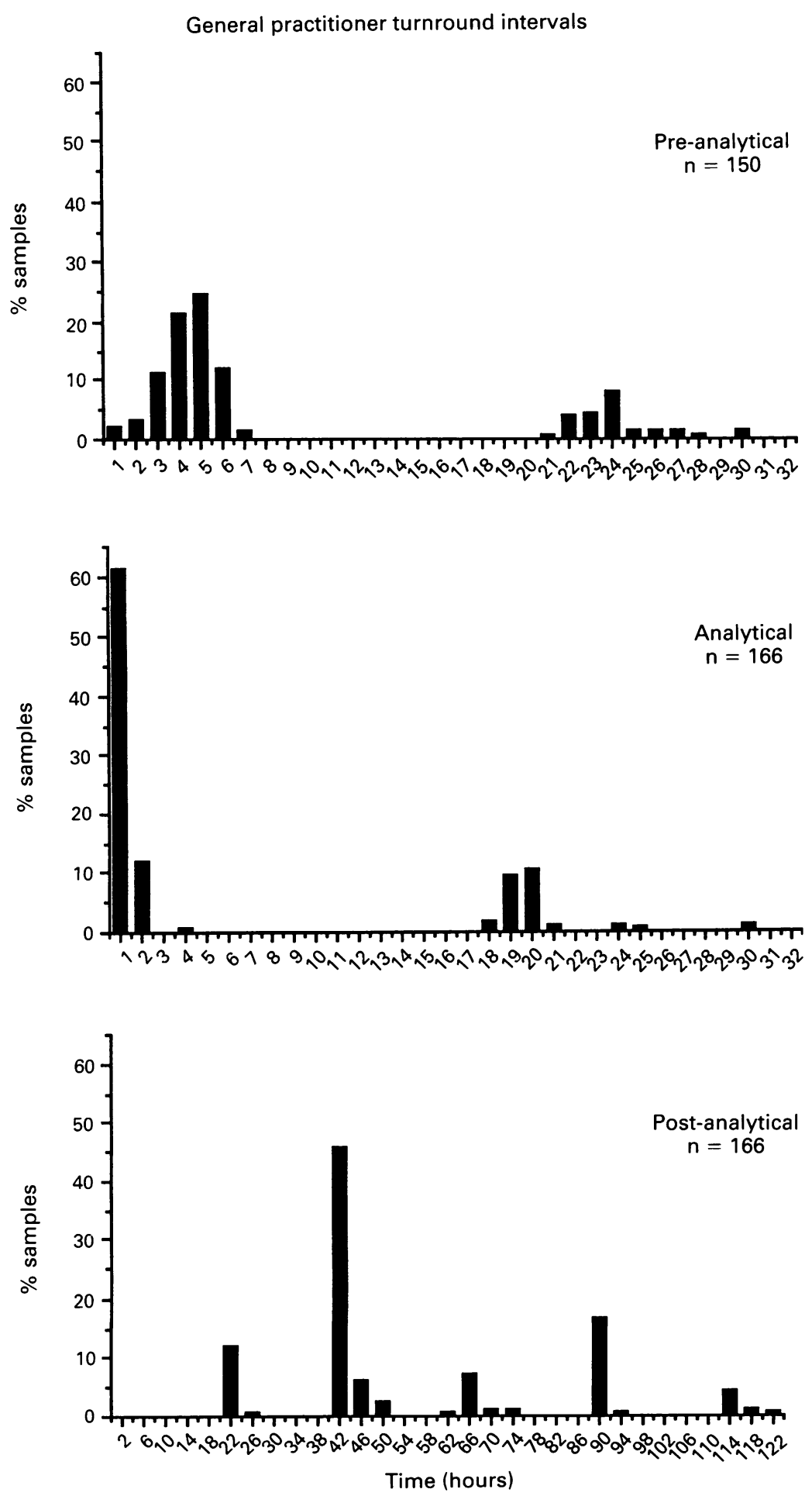

Figure 2 Distribution of the pre-, peri-, and post-analytical periods for samples from four general practices. recruited: one acute medical, one acute surgical, and one elective surgical. All employed clerical officers directly responsible for filing results, and were staffed by ward dedicated junior medical staff.

Outlying hospitals One satellite hospital was selected within the catchment area. This is one of the larger laboratory users outwith the Royal Infirmary, situated a distance of 1.5 miles through moderate city traffic from the department.

General practitioners Four practices were selected from within the catchment area: two large multipractice health centres and two single practice centres within 3 miles of the laboratory.

In all cases the proposed study design was discussed with clinical, nursing, and clerical staff involved in the specific sites, and consent obtained.

To assess the specific efficacy of the local Royal Mail links, two batches of 10 result envelopes were posted to one GP centre directly in a postbox at $12.00 \mathrm{pm}$ (for 12.30 $\mathrm{pm}$ collection) and at $5.00 \mathrm{pm}$ (for $8.00 \mathrm{pm}$ collection) on the same day. The arrival times of the 20 envelopes were recorded by the practice manager. This provided more specific figures than those which can be provided by the Post Office itself.

\section{Results}

Data from the 436 samples identified during this study are shown in the table. The turnround times from GP samples are shown in fig 2 .

The pre-analytical period varied considerably between the three sites; all satellite hospital samples with venesection times recorded were received within seven hours, as were $98 \%$ of Glasgow Royal Infirmary samples. However, only $76 \%$ of general practitioner samples arrived within seven hours, reflecting the number of samples withdrawn after the daily van collection. This generates a substantial number of results which are invalid due to sample age on arrival.

For the analytical time, a bimodal distribution was observed for both the satellite hospital and for general practitioner samples. The Glasgow Royal Infirmary samples demonstrated a single peak of analytical time with $97 \%$ of samples analysed on the day of receipt. Though the distribution was bimodal, $73 \%$ of general practitioner samples were analysed on the same day compared with less than $50 \%$ of the satellite hospital samples (importantly, the latter arrive in the laboratory later in the day).

In the post-analytical period the satellite hospital and general practitioners received their reports at 24 hour intervals after between one and five days, indicative of the once-a-day dispatch. The Glasgow Royal Infirmary wards received more than $70 \%$ of their reports within four hours of production.

All 20 result envelopes (for positive controls) were received at 8.30 am the following morning. This suggests an achievable post- 
analytical time by mail of between 12 and 20 hours, depending on times of posting and collection.

\section{Discussion}

Individual differences in laboratories are such that this study design may not be directly applicable to other laboratories. It does, however, establish a framework for planning and executing such a study and for solving a number of problems which will be common to all laboratories.

The principal resource behind the study is the laboratory's sophisticated record management system. In addition to recording date and time of sample withdrawal and receipt, we are able to record report production time on individual form types. This facility, however, only records the time of the first production of a report irrespective of whether it is complete or with some result(s) outstanding.

The sites involved in the study were considered as representative users of the laboratory service. They also had specific staff responsible for report receipt. One ward which was initially chosen, and subsequently found unsuitable, had a very rapid turnover of patients and was serviced by several junior medical staff. We believe that the success of this study is due in part to careful selection of target units, taking into consideration these difficulties.

While rounding the times to full hours may have caused some errors, the results clearly identify the major area of delay as post-analytical. A simple positive control confirmed that first class post directly from the laboratory would significantly decrease this post-analytical delay to distant sites.

In the absence of direct computer links, mail posted by the laboratory may serve as a quality standard against which any hospital transport improvements may be measured. Our laboratory has already approached the hospital administration about this problem.

The problem of the exceptionally long analytical time for the satellite hospital samples may be alleviated simply by arranging for earlier delivery of samples to the laboratory, improving patient care.

This study has investigated the components of turnround time and highlighted the areas where most delay is incurred. However, in providing a service to clinicians merely speeding up turnround is not sufficient. It is also important to discuss specific needs. This may involve changes from the timing of sample withdrawal through all stages of turnround to ensure that a result report is available when required-for example, in time for a consultant ward round. Changing work practices such as doctors' shifts and increased demand for rapid patient turnround may also require a change in timing of report receipt both in terms of direct patient care or as a factor in permitting early discharge of a patient.

This study demonstrates the potential for improvement in all stages of laboratory turnround times in general, and in the process of report distribution in particular. It also highlights the interrelation between turnround times, such as the impact of late arrival in the pre-analytical period on the analytical period itself. It demonstrates the major potential impact that simple changes such as moving van delivery times by one or two hours or direct mailing of reports could have on overall laboratory quality of service.

We are presently performing a more detailed analysis of on-site sample turnround times to identify any specific delays within the laboratory. We wish to audit new transporting systems such as a proposed vacuum transport system for samples and the development of electronic mail for the return of results to confirm the potential implication on pre- and post-analytical times. It will only be through such auditing that the chemical pathology laboratory can begin to address the difficulties in instituting some of the real time analysis protocols now being proposed.

We thank the members of Glasgow Royal Infirmary Laboratory Directorate Audit Committee (Chairman Professor F D Lee) for their advice in initiating this study, and in particular Dr F J Dryburgh for her advice during this study and in help with the manuscript.

1 Audit Commission. Working with users. In: Critical Pathology: An analysis of the pathology services. London HMSO, 1993:9-13.

2 Murphy P G, Crothers E. A simple and direct method of turnround time audit in a microbiology laboratory. $f$ Clin Pathol 1992;45:738-40.

3 Scottish Health Service Common Services Agency. Clinical Chemistry. In: Laboratory Statistics. Year endin 31 March 1991. Edinburgh: Information \& Statistics Division. Crown Copyright, 1991. 